BULL. AUSTRAL. MATH. SOC.

VOL. $2(1970), 283-284$.

\title{
On an infinite integral linear group
}

\author{
I. H. Farouqi
}

Let $A$ be a free abelian group of countably infinite rank and $\Gamma$ the automorphism group of $A$. This group $\Gamma$ is found to differ radically from the integral linear group of finite dimension in the "density" of the set of normal subgroups it contains.

The congruence subgroups $\Gamma(m)$ consisting of all automorphisms $\gamma$ such that $a r-a \in m A$ for all $a \in A$ are, of course, normal in $\Gamma$ for each positive integer $m$. Also, the finitary automorphisms, that is, those which act non-trivially only on some direct summand of $A$ of finite rank, form a normal subgroup $\Phi$ of $\Gamma$. Every normal subgroup of $\Gamma$ intersects $\Phi$ non-trivially, but the intersections $\Phi \cap \Gamma(m)$ are, essentially, the only normal subgroups of $\Phi$.

Other types of normal subgroups of $\Gamma$ arise from infinite descending chains of subgroups of $A$. Let $f: N \rightarrow N$ be a function defined on the set of positive integers such that $f(i)$ properly divides $f(i+1)$ for all $i$ and let $F$ be the set of all such functions. We define a subset $\Sigma(f)$ of $\Gamma$ as follows: $\sigma$ is an element of $\Sigma(f)$ if and oniy if there exists a descending chain of subgroups of $A$, namely, $A=H_{1} \geq H_{2} \geq \ldots \geq H_{i} \geq \ldots$ such that for all $i$ the rank $r(i)$ of $H_{i} / H_{i+1}$ is finite and $h_{i} \sigma-h_{i} \in f(i) A$ for $h_{i} \in H_{i} . \Sigma(f)$ is a normal subgroup of $\Gamma$ and there are uncountably many of these.

Moreover, each $\Sigma(f)$ contains many normal subgroup chains of the order type of the positive reals obtained as follows. Let $f^{*}: N \rightarrow R$ take non-negative real values. An element $\sigma$ of $\Sigma(f)$ belongs to $\Sigma\left(f, f^{*}\right)$ if and only if there is a positive real constant $c(\sigma)$ such

Received 19 November 1969. Thesis submitted to the Australian National University, June 1969. Degree approved, September 1969. Supervisors: Professor Hanna Neumann, Professor B.H. Neumann. 283 
that $r(i) \leq c(\sigma) f^{*}(i)$ for all $i$. Then $\Sigma\left(f, f^{*}\right)$ is a normal subgroup of $\Gamma$, and if $f^{*}, g^{*}$ and the quotient $f^{*} / g^{*}$ are monotonically increasing and unbounded, then $\Sigma\left(f, f^{*}\right)$ properly contains $\Sigma\left(f, g^{*}\right)$. Now take for example for each real $\alpha>0$ the functions given by $f_{\alpha}^{*}(i)=i^{\alpha}$ to confirm the claim made above. 